\title{
Tratamiento Temprano de la Maloclusión Clase III con aparatología Ortopédica: Reporte de caso con 7 años de control
}

\section{Resumen}

Una de las maloclusiones más complejas de diagnosticar y de tratar es la maloclusión clase III. Se caracteriza por una alteración en la relación sagital de los maxilares, ya sea por una deficiencia o retrusión maxilar o por un prognatismo o macrognatismo mandibular, o bien sea una combinación de las anteriores. La etiología es multifactorial y es debida a una interacción de factores hereditarios y ambientales.

Se presenta una paciente de 4 años de edad, sexo femenino, con patrón hereditario de clase III en su familia materna. Relación esquelética clase III con mandíbula aumentada en longitud, protruida, rotando posterior y maxilar superior re- truído. Tipo de crecimiento vertical, con un perfil recto y mordida cruzada anterior y posterior unilateral izquierda.

Se instauró tratamiento con aparatología ortopédica durante el periodo de dentición decidua hasta la dentición permanente, para lograr así, equilibrar el crecimiento maxilomandibular. Uno de los aspectos clínicos más complicados del manejo ortopédico de la maloclusión clase III es la recidiva después del período de tratamiento activo; sin embargo en nuestra paciente se consiguen cambios a nivel esquelético, dental y estéticos que se mantuvieron durante el tratamiento.

Palabras claves: maloclusión de Angle clase III, efectividad, aparatos activadores

Case report

\section{Early treatment of Class III malocclusion with orthopedic appliances: Case report with 7 years of control}

\begin{abstract}
One of the most difficult malocclusions to diagnose and treat is Class III malocclusion. It is characterized by an alteration in the sagittal re-

lationship of the jaws, either by a deficiency or maxillary retrusion or mandibular prognathism, or whether a combination of the above. The etiology is multifactorial and is due to an interaction of hereditary and environmental factors.

\footnotetext{
${ }^{1}$ OdontopediatrÍa y Ortodoncia Preventiva Universidad CES

${ }^{2}$ Director Posgrado Odontopediatría y Ortodoncia Preventiva, Universidad CES, Profesor Asistente de posgrado de Odontopediatría y Ortodoncia, Universidad CES

${ }^{3}$ Ortodoncista CES, Profesora Asistente de Posgrados de Odontopediatría y Ortodoncia, Universidad CES
} 
A 4-year-old female with hereditary pattern of class III malocclusion in his mother's family is presented. Skeletal Class III, increased jaw length, protruded, rotating posterior and maxilla retruded. Vertical growth type, straight profile, anterior crossbite and posterior left-sided. Orthopedic appliance therapy was instituted during the deciduous dentition to the permanent dentition, to achieve, balancing the maxilloman- dibular growth. One of the most complex aspects of orthopedic clinical management of Class III malocclusion is the possibility of relapse after active treatment period, however, in our patient changes in skeletal, dental and aesthetic aspects achieved during treatment have been stable.

Key words: malocclusion Angle class III, effectiveness, activador appliances.

Relato de caso

\section{Tratamento precoce da Má oclusão de Classe III com aparatologia ortopédica: relato de caso com 7 anos do controle}

\section{Resumo}

Uma das más oclusões mais complexas de diagnosticar e tratar é a má oclusão de Classe III. Caracteriza-se por uma alteração na relação sagital dos maxilares, seja por deficiência ou retrusão maxilar ou por prognatismo ou macrognatismo mandibular, ou ainda por uma combinação desses eventos. A etiologia é multifatorial e envolve uma interação de fatores hereditários e ambientais.

Este artigo apresenta o caso de uma paciente de 4 anos de idade, do sexo feminino, com padrão hereditário de classe III em sua família materna. Apresentava relação esquelética de classe III, protuída, com rotação posterior e maxila superior retruída. Crescimento vertical, com um perfil reto e mordida cruzada anterior e posterior unilateral esquerda.

Foi instituído tratamento com aparatologia ortopédica na dentição decídua o qual permaneceu até a dentição permanente, com objetivo de equilibrar o crescimento maxilomandibular.
Um dos aspectos clínicos mais complicados do manejo ortopédico da má oclusão de Classe III é a recidiva depois do período de tratamento ativo, entretanto, nesse paciente foram conseguidas modificações à nível esquelético, dentais e estéticos durante o tratamento que se mantiveram estáveis.

Palavras chave: Má oclusão de Angle Classe III, Efetividade, aparelhos ativadores.

\section{Introducción}

La maloclusión clase III esquelética está asociada a una desviación en la relación sagital del maxilar y la mandíbula, caracterizada por alteración de un solo componente o una combinación de las siguientes variables: la mandíbula puede tener una longitud aumentada con respecto al maxilar o tener un exceso de crecimiento; el maxilar puede ser más pequeño con respecto a la mandíbula o tener una deficiencia de crecimiento; el maxilar puede estar en una posición retruida con respecto a la mandíbula; 
la mandíbula puede estar posicionada más adelante que el maxilar o puede existir una rotación mandibular hacia adelante causando una proyección del mentón horizontalmente con una posición más prognática. $(1,2)$

La etiología se asocia con genética, factores ambientales y hábitos, aunque algunos autores consideran las influencias funcionales como secundarias o adaptativas. Entre los factores ambientales que se han reportado se encuentran: la respiración

oral, unas amígdalas hipertróficas, defectos anatómicos congénitos, alteraciones hormonales, hábito de protrusión lingual, trauma y erupción irregular de los incisivos permanentes, entre otros.(2-4) En cuanto a la herencia, un estudio realizado por Gorlin y colaboradores en 1965, informaron que el prognatismo estaba asociado con ciertas condiciones del cromosoma $\mathrm{X}$ aneuploidal, afirmando que el prognatismo incrementa con cada cromosoma $X$ adicionado, mostrando mayor prognatismo la mandíbula. $(5,6)$

La incidencia de esta maloclusión en la población blanca ha sido con un porcentaje de $1 \%$ a $5 \%$, en la población asiática aumenta la incidencia de $9 \%$ a $19 \%$ y en la población latina la incidencia es aproximadamente del 5\%. (3) Un estudio realizado por Thilander y colaboradores en el 2001, encontró en 4724 niños y adolescentes Bogotanos, un $3.7 \%$ de prevalencia de maloclusión clase III en cuanto a la relación molar de mesioclusión y, un overjet menor a cero (0) en el $5.8 \%$. De un total de 1441 niños, el 4,9\% se asoció a mesioclusión en dentición permanente, y de 1539 pacientes en dentición mixta temprana, un $3.9 \%$ se asoció a mesioclusión (7).

En la clínica, las maloclusiones clase III se consideran entre los casos más difíciles a tratar y la intervención temprana es indicada para obtener una relación normal entre el maxilar y la mandíbula. La finalidad es la eliminación de factores que puedan inhibir el crecimiento anterior del hueso maxilar y redirijan el crecimiento mandibular; como las mordidas cruzadas anteriores. Se han desarrollado varios aparatos ortopédicos para la corrección de estos casos, incluyendo aparatos de protracción maxilar, aparatos funcionales, y la mentonera. (8)

En estudios recientes sobre los efectos a largo plazo de la terapia de protracción maxilar con máscara facial y expansión rápida palatina, parecen indicar que los resultados del tratamiento ortopédico de las maloclusiones clase III es favorable cuando se inicia antes de que el pico de crecimiento puberal, además un promedio de tres cuartas partes de los pacientes clase III que reciben tratamiento ortopédico mantienen un overjet positivo después de la maduración esquelética pos puberal $(9,10)$.

Ensayos clínicos realizados donde comparan los cambios ortopédicos de los pacientes con maloclusión Clase III tratados versus el crecimiento natural de los controles clase III no tratados, concluyen y confirman que las intervenciones ortopédicas son eficaces y efectivas en la corrección de discrepancias esqueléticas. (11-13)

Los efectos a largo plazo de la corrección de la clase III se deben evaluar cuando los pacientes han alcanzado la edad ósea del estirón pos puberal. E independientemente de la opción de tratamiento, un resultado funcional y estético que sea estable a largo plazo es el resultado deseado. Por otra parte, se reporta un aumento en la autoestima de los pacientes y satisfacción de los padres, que está relacionada con la rápida mejoría de la estética facial generalmente obser- 
vada después de la intervención ortopédica en pacientes jóvenes con maloclusión clase III. (14)

El objetivo de este artículo es presentar un caso clínico donde se muestra la efectividad en la corrección de una maloclusión clase III con intervención temprana, evitando consecuencias desfavorables a través del crecimiento, y logrando una adecuada estabilidad oclusal y esquelética.

\section{Reporte de caso}

Paciente de 4 años de edad, sexo femenino, quien llegó a consulta de odontopediatria en la
Clínica CES Sabaneta en marzo de 2006, traída por su madre, con motivo de consulta: "el crecimiento de la mandíbula de mi hija".

Al momento de la consulta no presenta enfermedades sistémicas, se reporta antecedentes de clase III en otros miembros de la familia incluyendo a la madre.

Entre sus antecedentes odontológicos solo ha tenido tratamiento de operatoria.

Al examen clínico facial se observa una paciente con perfil recto, malar poco expresivo, tercios y quintos simétricos, competencia labial y labio inferior protruido (figura $1 \mathrm{~A}-\mathrm{C}$ ).
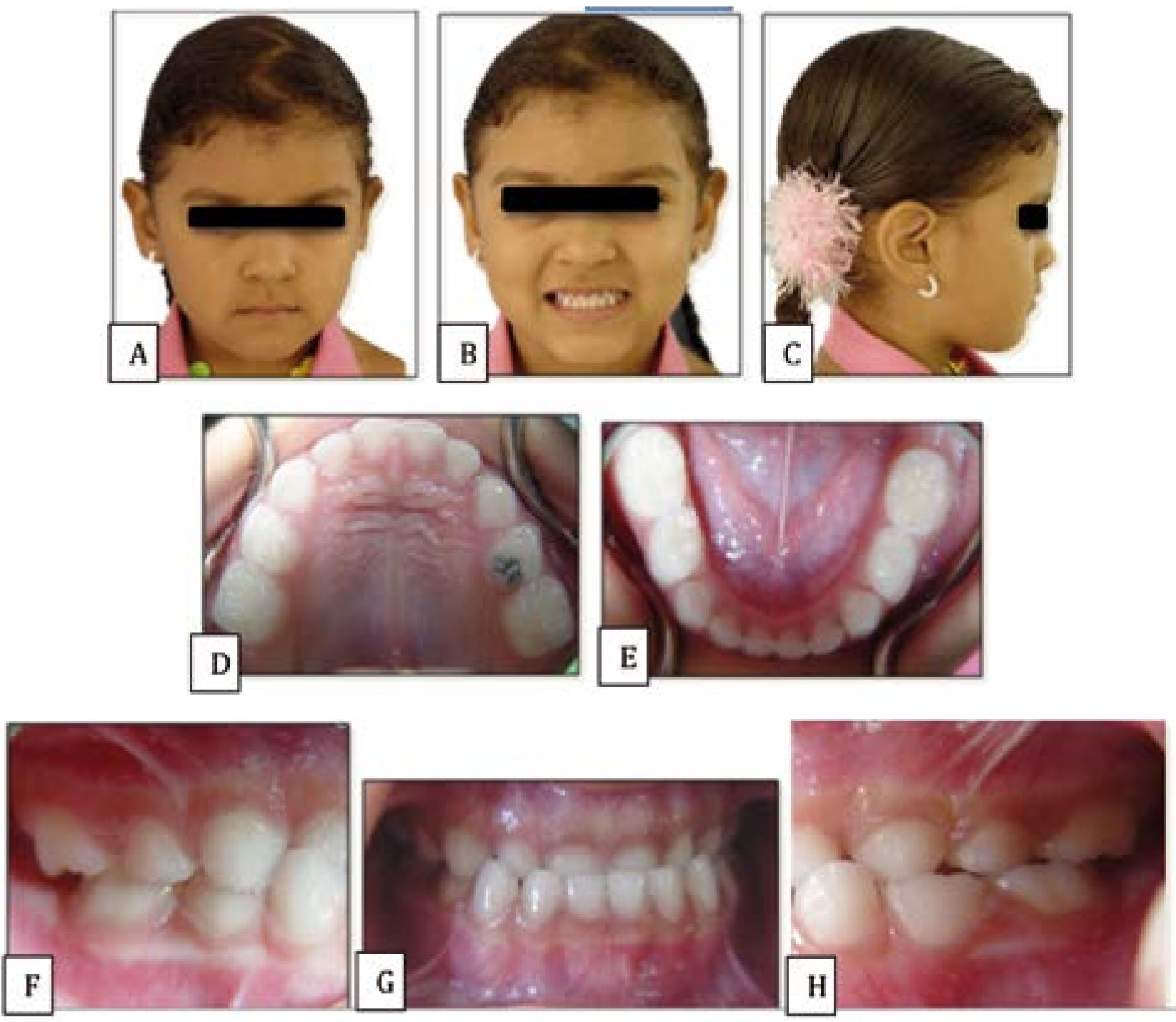

Figura 1. Fotografías pre tratamiento extraorales, frente, sonrisa, perfil $(A-C)$ e intraorales $(D-H)$. 

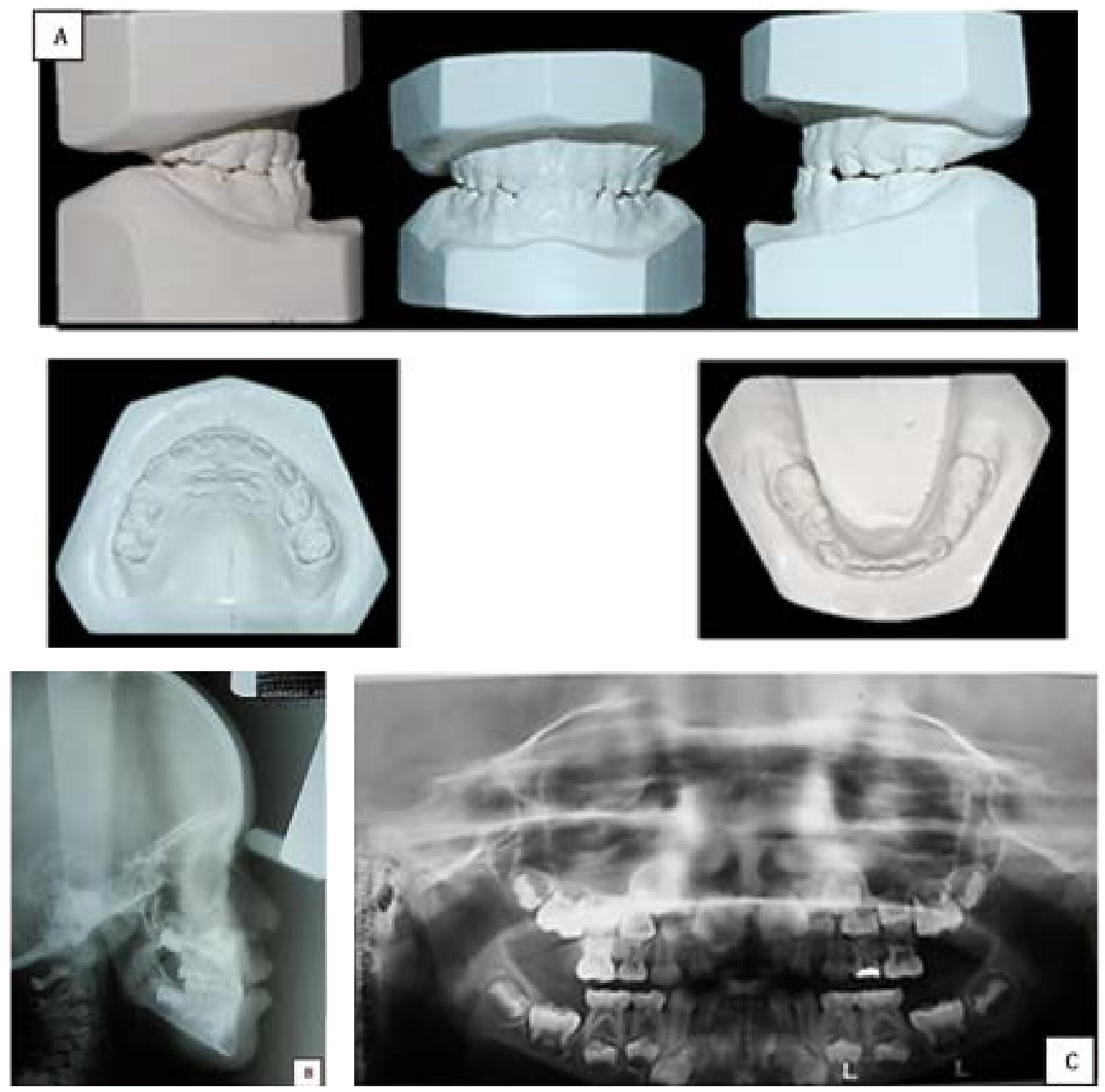

Figura 2. Modelos pre tratamiento (A), radiografías inicial cefálica lateral (B) y panorámica (C).

Intraoralmente, se observa dentición decidua, escalón mesial derecho e izquierdo, mordida cruzada anterior y posterior unilateral izquierda (Figura 1D-H).

\section{Ayudas diagnósticas}

- Modelos de estudio: arco superior e inferior ovalados, sin asimetrías en sentido anteroposterior ni transversal. En máxima intercuspidación presenta overjet $-2 \mathrm{~mm}$, overbite $-50 \%$, mordida cruzada entre 52 83/52-82-81/62-71-72/ 62-73/63-74/65-75, línea media $0.5 \mathrm{~mm}$ desviada a la izquierda (Figura 2A).

- Radiografía cefálica: Relación esquelética clase III, con mandíbula aumentada en longitud, protruida, rotando posterior, maxilar retruído, tipo de crecimiento vertical, incisivo superior vestibularizado (Figura 2B).

- Radiografía panorámica: dentición decidua, secuencia de erupción de permanentes adecuada, desarrollo radicular normal, y estruc- 


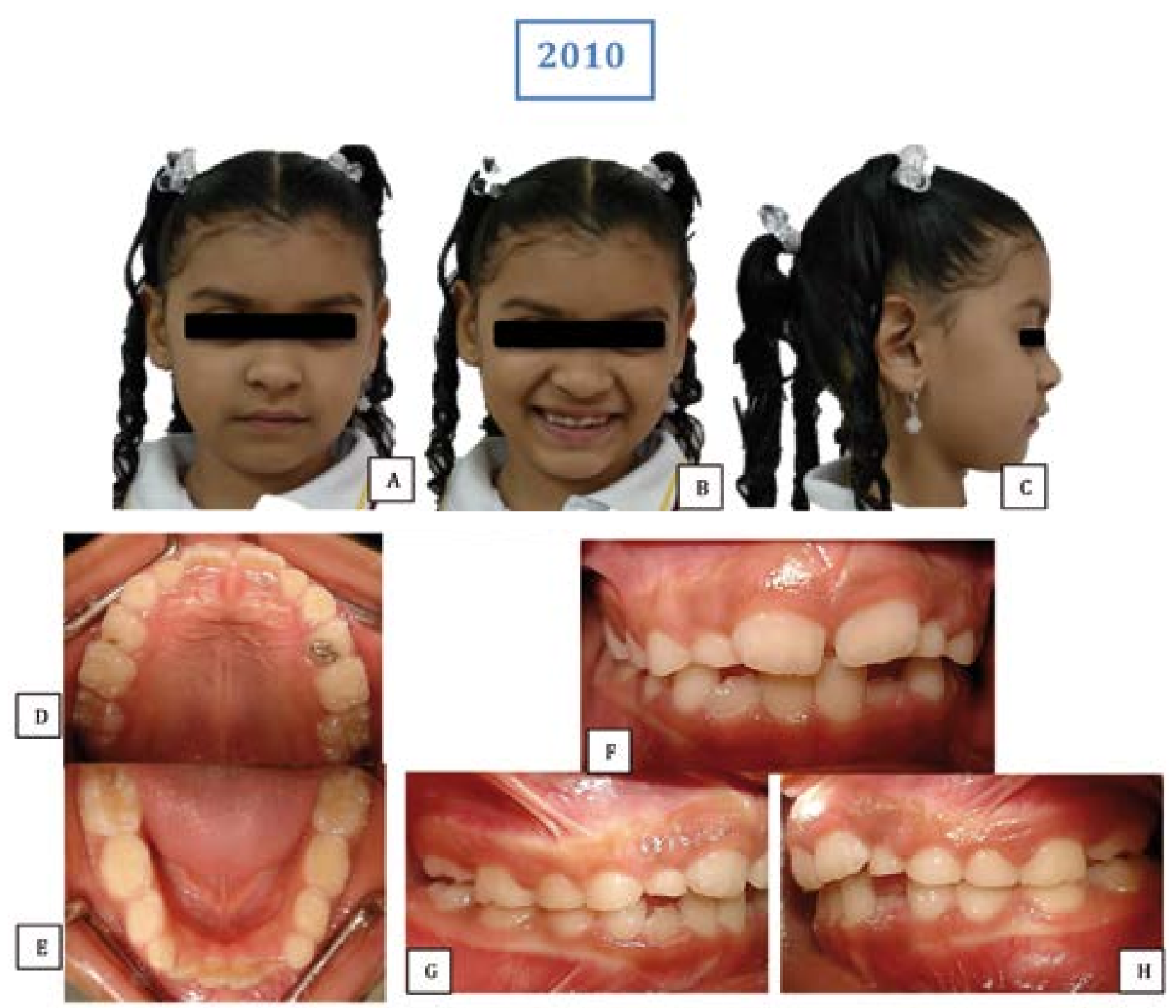

Figura 3. Fotografias extraorales (A-C) e intraorales (D-H) intermedias 2010.

turas óseas normales, no se observan ausencias de gérmenes dentales (Figura 2C).

- Análisis funcional: masticación bilateral alternada, no presentaba hábitos, ni alteraciones funcionales.

- Objetivos del tratamiento: Corregir mordida cruzada anterior y posterior unilateral izquierda, corregir relación esquelética clase III, corregir overjet y overbite, mejorar el perfil, controlar el crecimiento vertical, estabilidad del tratamiento.

\section{$\underline{\text { Fases del tratamiento }}$}

- Fase I: 2006/ 4 años: inicialmente se realiza el tratamiento preventivo, posteriormente se descruza mordida para evitar y eliminar una limitación del crecimiento normal del maxilar, se corrige overjet negativo por un overjet de $2 \mathrm{~mm}$. Esto se logra con la instalación de Adams Porter y plano inclinado anterior, 4 meses después se instala mentonera vertical para control de rotación posterior mandibular. 
- Fase II: 2008 / 6 años: se realiza una reevaluación 2 años después, donde se encuentra un maxilar hipoplasico, la mandíbula continúa aumentada en longitud y rotando posterior; en este momento se decide instalar máscara facial con tornillo Hyrax. Se inicia con una activación del tornillo 1 / 4 de vuelta diario por 8 días, posteriormente se instala máscara facial de protracción con uso diario de 14 horas y elásticos a $30^{\circ}$ con respecto al plano oclusal, la cual fue utilizada por 2 años.

- Fase III: 2010 / 8 años (figura 3): continua con un overjet de $2 \mathrm{~mm}$ entre 11 y 41, relación

\section{3}
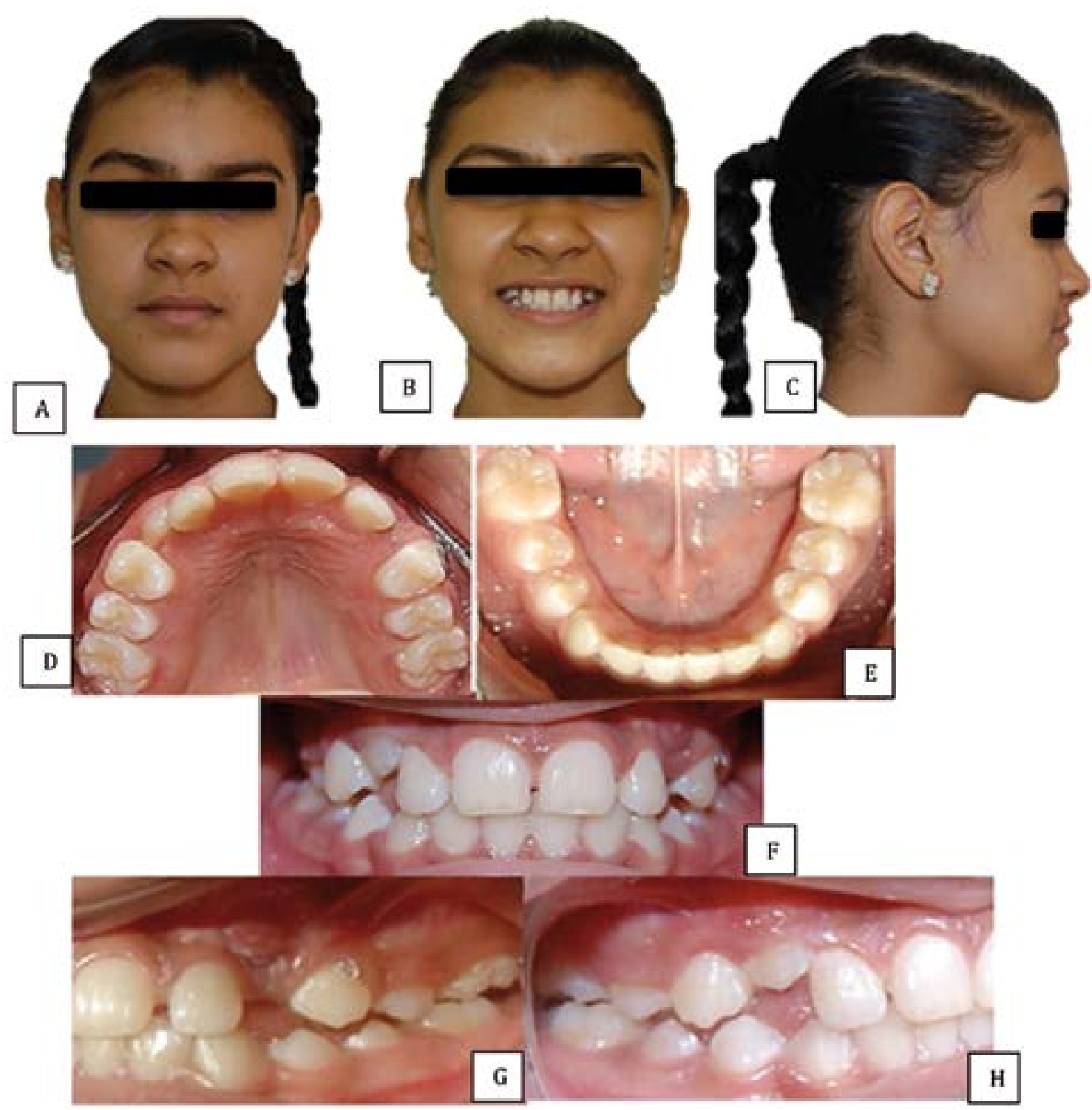

Figura 4. Fotografías extraorales ( $A-C)$ e intraorales (D-H) 7 años después de iniciar tratamiento ortopédico -2013. 

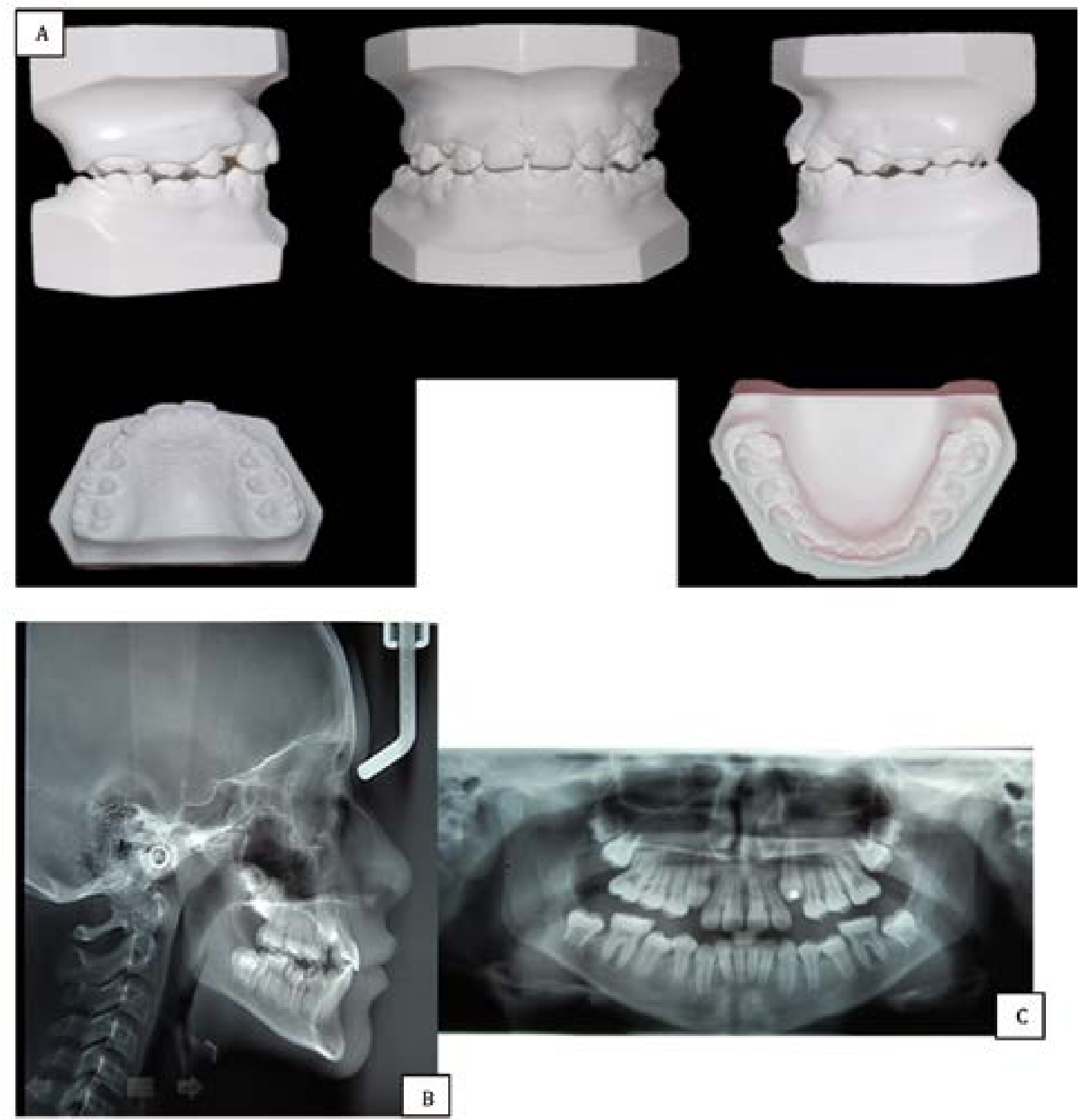

Figura 5. Ayudas diagnosticas: modelos de estudio (A), radiografía cefálica (B) y panorámica (C) 7 años después (2013).

intermaxilar esquelética clase I con maxilar superior hipoplasico y protruido, mandíbula de buen tamaño, bien posicionada y rotando posterior. Se instala Klammt I para continuar retención del crecimiento, alineación de arcos y malposiciones dentarias.

- Fase IV 2012 / 10 años: 2 años después se observan 13 y 23 con riesgo de impactación, y sin espacio para su erupción, se decide instalar péndulo para distalizar molares y paralelamente se realizó tracción ortodontica de caninos. En el arco inferior se inicia guía de erupción y se instala arco lingual. Cuando se logra la distalización necesaria, y una posición vertical de caninos, se instala botón palatino como retención. 
Para octubre de 2013, con 11 años de edad, se encuentra con maloclusión clase I, línea media que coincide, overbite: $44 \%$, overjet: $3 \mathrm{~mm}$ entre $11 / 41$, arco superior e inferior ovalado, relación intermaxilar esquelética clase I con hipoplasia maxilar, mandíbula levemente protruida y rotando neutra según el análisis cefalometrico. En el análisis de maduración de vértebras cervicales, se encuentra en Cs4, es decir que el pico de crecimiento posiblemente inicio 1 o 2 años antes. En esta fase, se observa una marcada mejoría tanto en la armonía facial como en la oclusión. (Figuras 4 y 5 )
Finalmente se logran los siguientes cambios: se corrige mordida cruzada anterior y posterior, se logra una mejoría e overjet que pasa de $-2 \mathrm{~mm}$ a $3 \mathrm{~mm}$, corrección de la relación intermaxilar esquelética clase III, relaciones molares clase I, líneas medias coincidentes, mejoría en forma de arco y amplitud de los arcos, mejoría en la ubicación de 13 y 23, equilibrio del crecimiento maxilomandibular y estabilidad del tratamiento, evitando posible corrección quirúrgica (Figuras 5 y 6).

Los Cambios esqueléticos logrados se debieron principalmente a una corrección de la
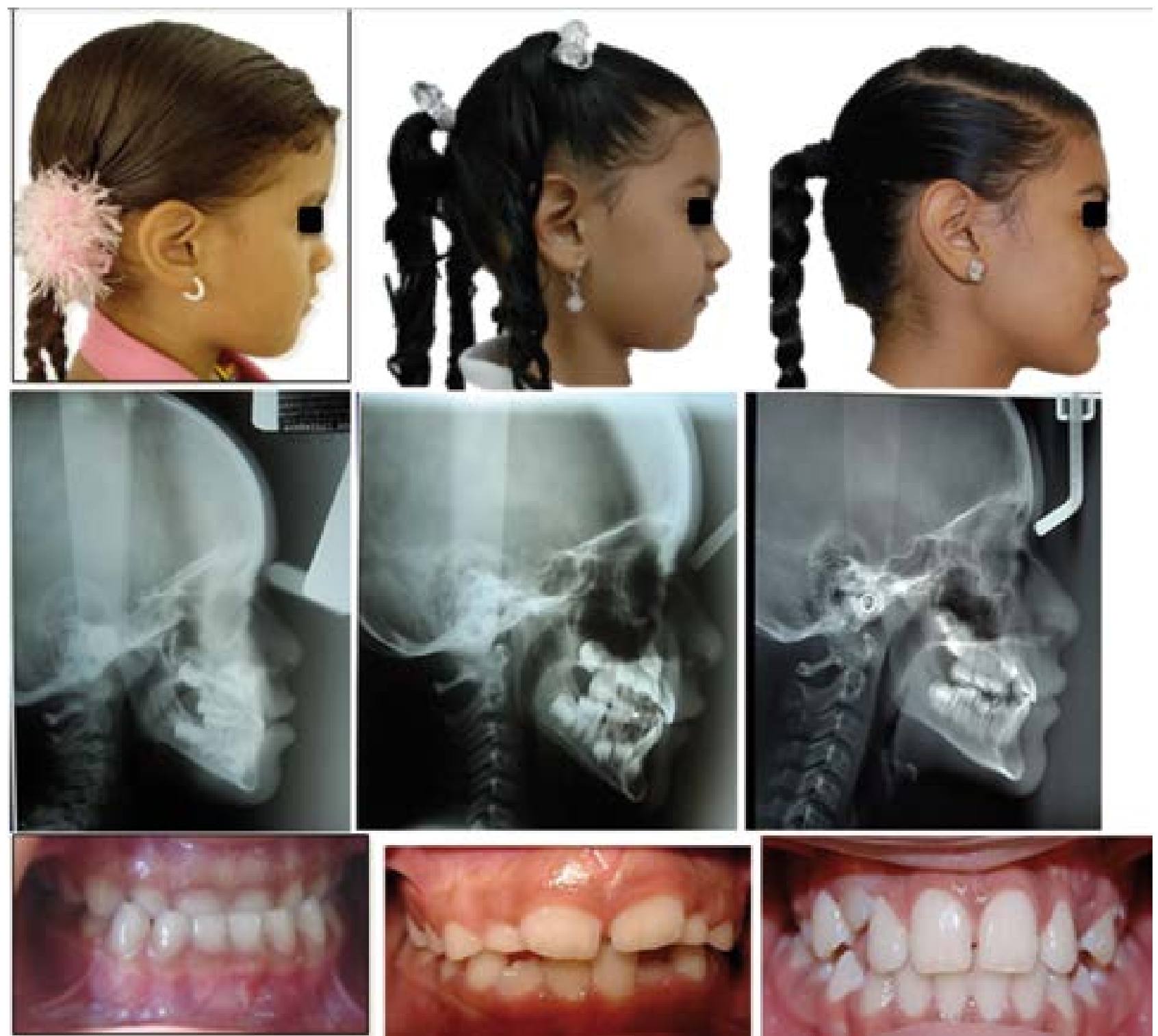

Figura 6. Perfil y oclusión antes y después de tratamiento ortopédico. 
Tabla 1. Valores cefalometricos iniciales, intermedios y finales.

\begin{tabular}{|c|c|c|c|}
\hline $\begin{array}{c}\text { Variables } \\
\text { cefalometicas }\end{array}$ & Iniciales 2006 & Intermedias 2010 & Finales 2013 \\
\hline CO-A & 74 & 83 & 83 \\
\hline SNA & $79.5^{*}$ & $84^{*}$ & $84^{*}$ \\
\hline Plo Af Mcliamara & -4.5 & 0.5 & 2 \\
\hline SNB & $80^{\circ}$ & $77.5^{\circ}$ & $82^{*}$ \\
\hline $\mathrm{Co} \cdot \mathrm{Gn}$ & 98 & 104 & 107 \\
\hline Pog/ MeNiamara & 8 & 10 & 5 \\
\hline Angulo goniaco & $141^{*}$ & $132^{*}$ & $130^{\circ}$ \\
\hline ENA - Mn & 57 & 62 & 58 \\
\hline ANB & $0^{\circ}$ & $75^{\circ}$ & $2^{*}$ \\
\hline Wits & -2 & 25 & 3 \\
\hline $\begin{array}{l}\text { Incisivo superiorl plano } \\
\text { palatal }\end{array}$ & $100^{\circ}$ & $115^{\circ}$ & $121^{*}$ \\
\hline $\begin{array}{l}\text { Ineistvo inferiorl plano } \\
\text { mandibular }\end{array}$ & $87^{*}$ & $925^{\circ}$ & $91^{*}$ \\
\hline
\end{tabular}

posición sagital del maxilar y la mandíbula (Tabla 1).

Al comparar la radiografía cefálica inicial y final de la paciente, se observa: una rotación aparente mandibular en sentido anterior, una rotación verdadera del maxilar en sentido posterior, con reabsorción en piso nasal, extrusión del molar superior y vestibularización del incisivo superior (Figura 7).
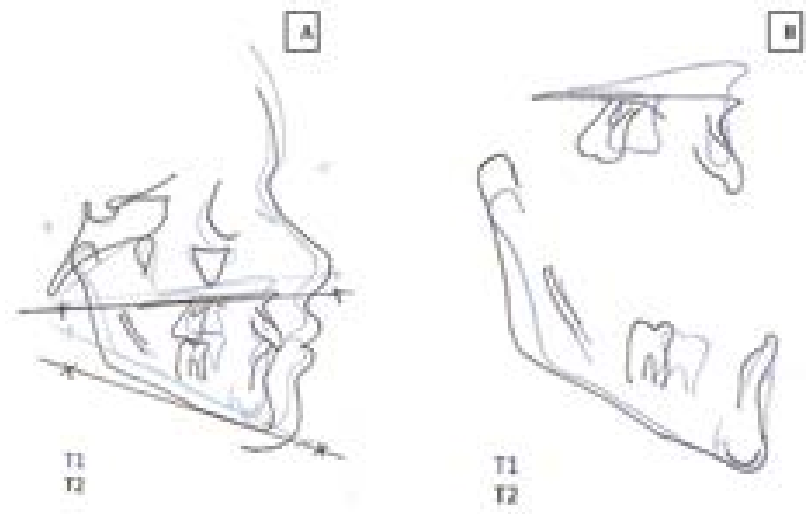

Figura 7. Superposición general donde se muestra rotación aparente $(A)$ y superposición maxilar y mandibular donde se observa rotación verdadera de cefalometría inicial (T1) y final (T2).
En la rotación verdadera mandibular se observa aposición en superficie posterior de rama, reabsorción en zona anterior a nivel de los incisivos, aumento del crecimiento anteroposterior y vertical del cóndilo lo que podría decir que cambio el patrón de rotación posterior a una rotación más anterior y control vertical del molar inferior.

\section{Discusión}

La maloclusión clase III generalmente se manifiesta desde una edad muy temprana, pero con respecto al momento óptimo para el tratamiento ortopédico, existe desacuerdo.

Numerosos estudios en la literatura han apoyado el tratamiento temprano para maximizar el avance anterior del maxilar y minimizar los efectos dentoalveolares $(15-17,11)$.

En cuanto a esos cambios esqueléticos encontrados en nuestra paciente (Tabla 1) se observa que el 
Tabla 2. Medida que demuestra las diferencias en posición del maxilar a los 4 y a los 11 años de la paciente, comparado con el crecimiento normal de pacientes clase III a los 11 años de edad.

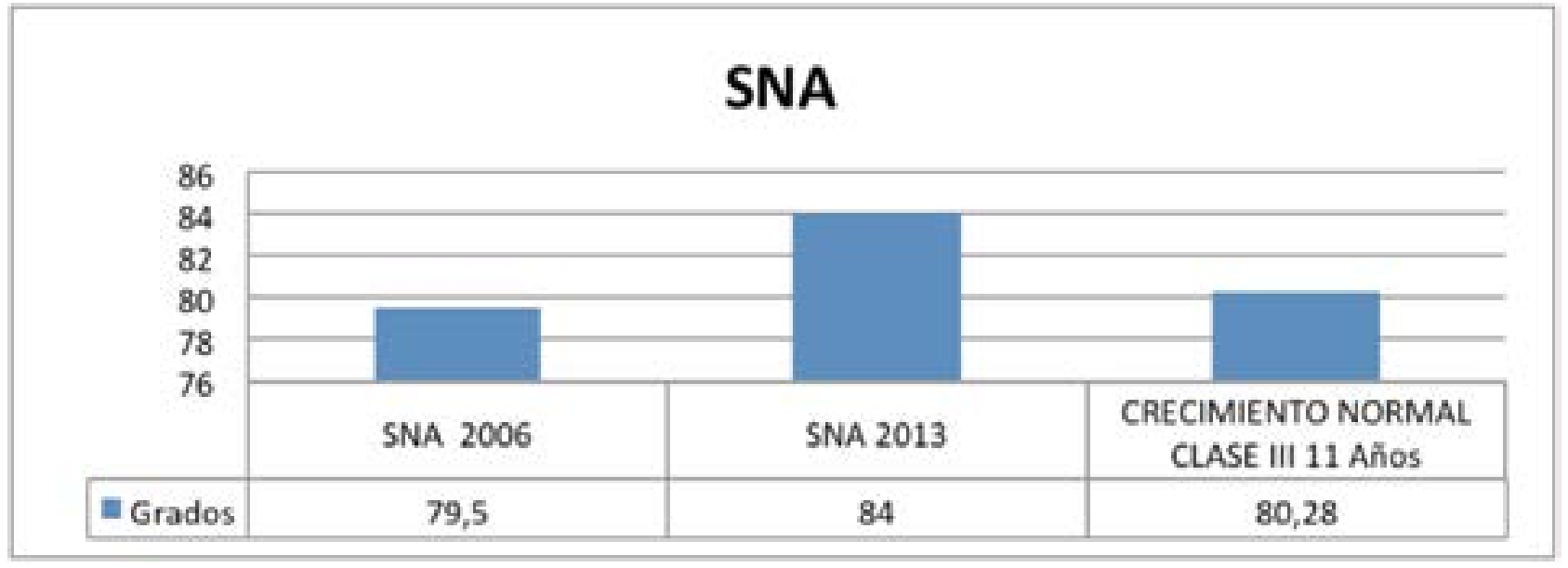

Tabla 3. Medida que demuestra las diferencias en longitud mandibular a los 4 y a los 11 años de la paciente, comparado con el crecimiento normal de pacientes clase III a los 11 años de edad.

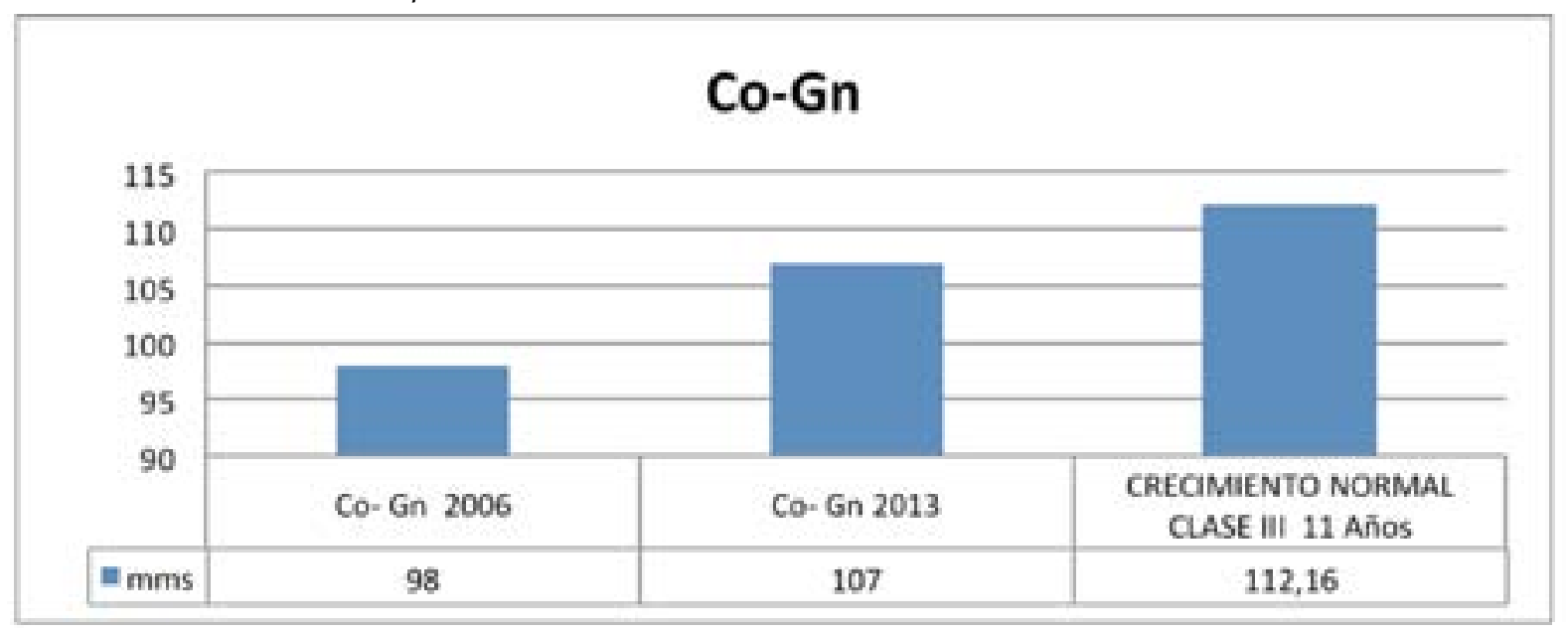

ángulo SNA aumenta del 2006 al 2010 mostrando un adelantamiento del maxilar atribuido al tratamiento, que se mantiene estable hasta el 2013 (Tabla 2). Pto A/ McNamara pasa de $-4.5 \mathrm{~mm}$ en 2006 a $0.5 \mathrm{~mm}$ en 2010 y aumenta a $2 \mathrm{~mm}$ en el 2013 .

Según el estudio de Bushang publicado en el 2011, en contraste con los pacientes clase I, la mandíbula de los pacientes clase III es más hiperdivergente y más grande. (19) Similar a lo encontrado en este caso clínico, donde la distancia Co-Gn aumenta, demostrando un crecimiento mandibular progresivo, con una ángulo goniaco estable entre el 2010 y el 2013 (Tabla 3).
La relación anteroposterior de los pacientes clase III empeora entre los 6 y 16 años de edad. En comparación con pacientes clase I, los pacientes clase III tienen ángulos SNB más grandes y ANB más pequeños. (19) Además se encuentra una disminución en el Wits con el crecimiento, lo que indica un agravamiento de la maloclusión clase III. (19) En la paciente se logra que el Wits aumente y el ANB permanezca estable durante el tratamiento (Tabla 4 y 5).

En cuanto al éxito y efectividad del tratamiento, una revisión sistemática realizada por Laura De Toffol y colaboradores en el 2008, donde evalua- 
Tabla 4. Medida que demuestra las diferencias del Wits a los 4 y a los 11 años de la paciente, comparado con el crecimiento normal de pacientes clase III a los 11 años de edad.

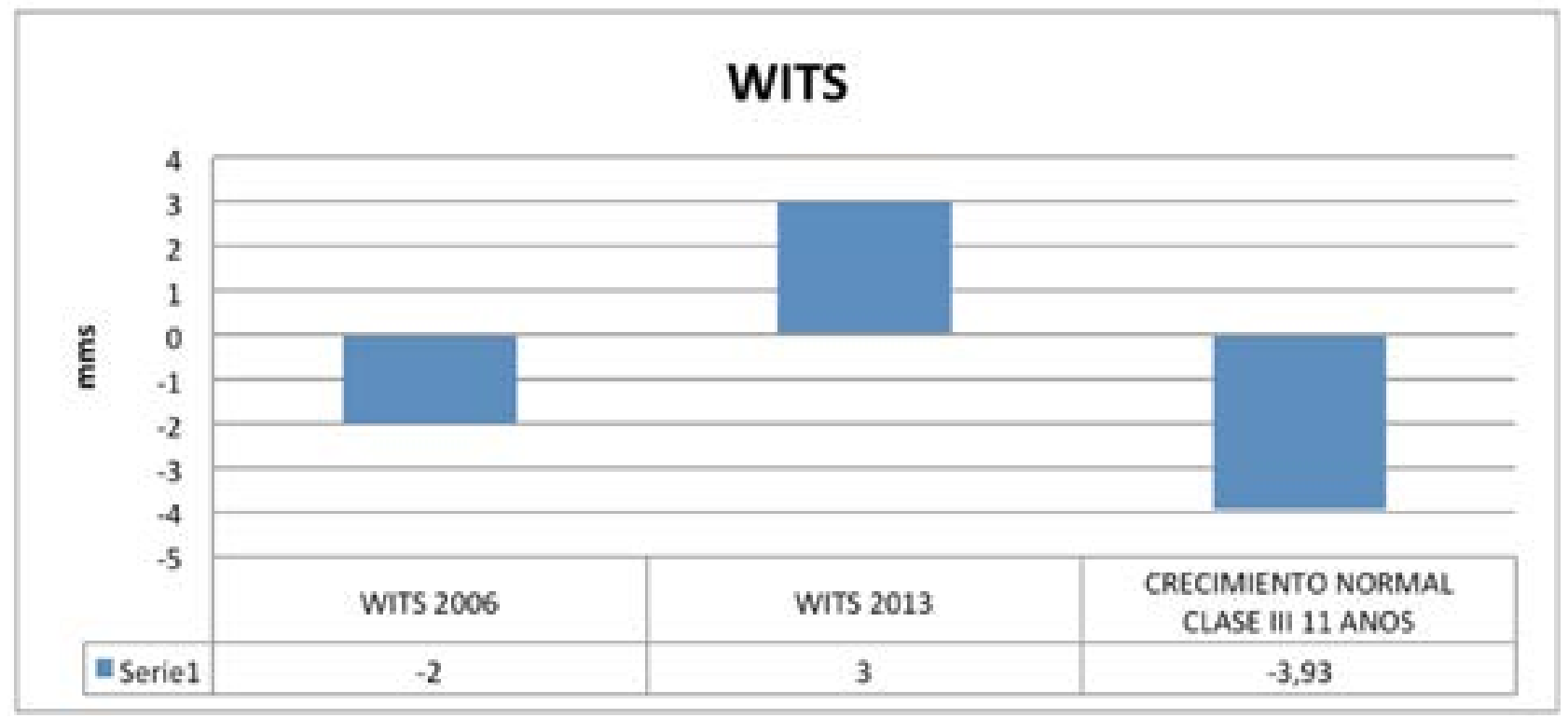

Tabla 5. Medida que demuestra las diferencias en la relación maxilomandibular a los 4 y a los 11 años de la paciente, comparado con el crecimiento normal de pacientes clase III a los 11 años de edad.

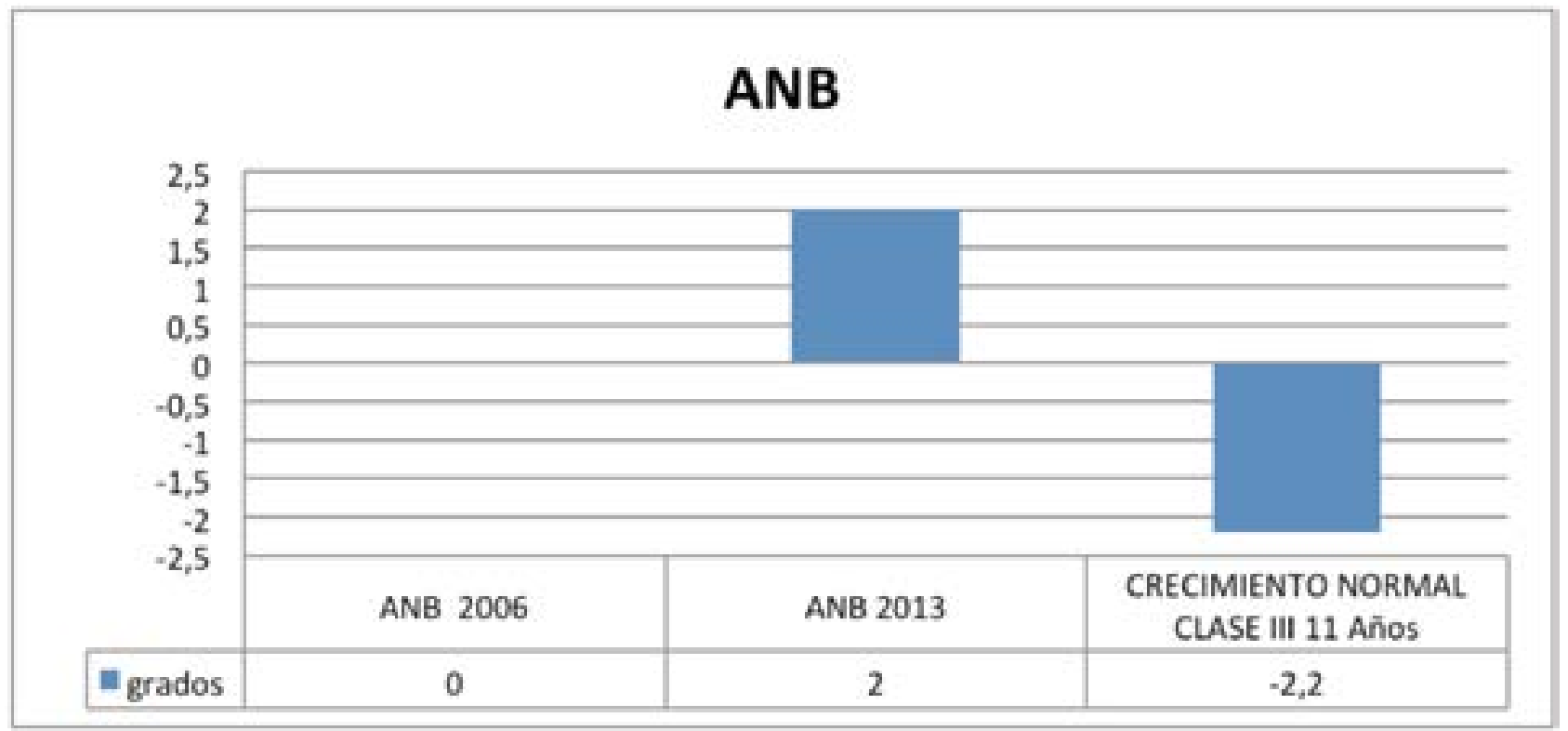

ron la evidencia científica sobre la efectividad del tratamiento ortopédico temprano en pacientes clase III, encontraron que de los artículos seleccionados, 5 informaron de una tasa de éxito del 100\% (20-24) mientras que en otro estudio reportaron el $85 \%$ de éxito. (25)

En un estudio realizado por Lorenzo franchi y Col en 2011, donde evaluaron los efectos a largo plazo de pacientes clase III tratados con mascara facial y expansión, se encontró que más del $70 \%$ de los pacientes (16 de 22) se podría considerar con éxito clínico a largo plazo, mientras que menos del 30\% de ellos (6 de 22) no tuvieron éxito 2 años después, debido a la recidiva en las relaciones oclusales. La tasa de prevalencia para el éxito a largo plazo del tratamiento de la 
maloclusión clase III con expansión y mascara aparece como un resultado favorable, y es similar a las tasas de éxito reportados en otros estudios sobre el tratamiento ortopédico de clase III que incluyeron un intervalo de tiempo después del tratamiento (el 75\% de éxito de acuerdo con Ngan y colaboradores). $(9,19,26)$

Westwood en el 2003 examinó 34 pacientes tratados con expansión rápida maxilar y máscara facial, antes del pico de crecimiento (8 años), seguidos de aparatología fija y evaluados 5 años y medio después del tratamiento. El crecimiento se comparó con una muestra control clase III sin tratar. Los resultados mostraron que el overjet y la relación molar del grupo tratado con clase III mejoraron de 3,9 a 4,5 mm, mientras que el grupo control clase III se agravó por $3 \mathrm{~mm}$. La corrección se debió principalmente a la restricción mandibular $(-2,5 \mathrm{~mm})$ y al avance maxilar (1,6 mm). En la etapa de observación final, 26 de los 34 pacientes $(76 \%)$ en el grupo tratado tuvo overjet positivo y 3 pacientes (9\%) tenían una relación incisal borde a borde. Los pacientes con overjet positivos de 4 a $5 \mathrm{~mm}$ o mayores durante la fase de tratamiento ortopédico generalmente pudieron mantener resultados favorables a largo plazo. (9) El overjet de nuestra paciente mejo- ro de $-2.0 \mathrm{~mm}$ en el 2006 a $3 \mathrm{~mm}$ hasta el 2013, lo que podríamos considerar como exitoso.

A pesar de los buenos resultados obtenidos en nuestra paciente, se requiere observación a largo plazo para asegurar la estabilidad y el éxito de un tratamiento ortopédico de manera temprana en pacientes clase III, como este aplicado.

\section{Conclusiones}

- Los cambios ortopédicos de los pacientes con maloclusión Clase III tratados versus el crecimiento natural de los controles clase III no tratados, concluyen y confirman que las intervenciones ortopédicas son eficaces y efectivas.

- El tratamiento temprano con fuerzas ortopédicas para avanzar el maxilar superior podría en algunos casos, reducir por completo la necesidad de intervención quirúrgica posterior.

- Los pacientes que reciben tratamiento ortopédico temprano tendrían una expectativa razonable de mejora de la autoestima, con estabilidad duradera, mientras que otros podrían ser tratados más tarde por una combinación de un tratamiento de ortodoncia y cirugía ortognática.

\section{Referencias}

1. Jacobson A, Evans WG, Preston CB, Sadowsky PL. Mandibular prognathism. Am J Orthod. $1974 ; 66$ (2):140-71.

2. Litton SF, Ackermann LV, Isaacson R, Shapiro B. A genetic study of class III malocclusion. Am J Orthod. 1970; 58:565 -77 .

3. Toffol LD, Pavoni C, Baccetti T, Franchi L, Cozza P. Orthopedic treatment outcomes in Class III malocclusion. A systematic review. Angle Orthod. 2008; 78(3):561-73.

4. Pascoe JJ, Hayward Jr, Costich Er. Mandibular prognathism: its etiology and a classification. J Oral Surg Anesth Hosp Dent Serv. 1960; 18:21-4. 
5. Gorlin RJ, Redman RS, Shapiro BL. effect of x-chromosome aneuploidy on jaw growth. J. Dent. Res. 1965; 44: SUPPL: 269-82.

6. Guyer EC, Ellis E III, McNamara, JA Jr, Behrents RG. Components of Class III malocclusion in juveniles and adolescents. Angle Orthod. 1986; 56:7-30.

7. Thilander B, Pena L, Infante C, Parada SS, de Mayorga C. Prevalence of malocclusion and orthodontic treatment need in children and adolescents in Bogota, Colombia. An epidemiological study related to different stages of dental development. Eur J Orthod. 2001; 23(2):153-67.

8. Abdelnabya Y; Nassarb E. Chin cup effects using two different force magnitudes in the management of lass III malocclusions. Angle Orthod. 2010; 80:957-62.

9. Westwood PV, McNamara JA Jr, Baccetti T, Franchi L, Sarver DM. Long-term effects of Class III treatment with rapid maxillary expansion and facemask therapy followed by fixed appliances. Am J Orthod Dentofacial Orthop. 2003; 123:306-20.

10. Baccetti T, Franchi L, McNamara J. Cephalometric variables predicting the longterm success or failure of combined rapid maxillary expansion and facial mask therapy. Am J Orthod Dentofacial Orthop. 2004; 126(1):16-22.

11. Baccetti T, Franchi L, McNamara Ja Jr Treatment and posttreatment craniofacial changes after rapid maxillary expansion and facemask therapy. Am J Orthod Dentofacial Orthop. 2000; 118: 404-413.

12. Macdonald K E, Kapust A J, Turley P K. Cephalometric changes after the correction of Class III malocclusion with maxillary expansion/ facemask therapy. Am J Orthod Dentofacial Orthop . 1999; 116: 13-24.

13. Tortop T, Keykubat A, Yüksel S. Facemask therapy with and without expansion. Am J Orthod Dentofacial Orthop. 2007; 132: 467-474.

14. Chen LL. The effects of maxillary protraction and its long-term stability-a clinical trial in Chinese adolescents. Eur J Orthod. 2012; 34(1):88-95.

15. Takada K, Petdachai S, Sakuda M. Changes in dentofacial morphology in skeletal Class III children treated by a modified maxillary protraction headgear and a chin cup: a longitudinal cephalometric appraisal. Eur J Orthod. 1993; 15: $211-221$.

16. Chong Y H, Ive J C, Årtun J. Changes following the use of protraction headgear for early correction of Class III malocclusion. Angle Orthod. 1996; 66: 351-362.

17. Shanker $\mathrm{S}$ et al. Cephalometric A-point changes during and after maxillary protraction and expansion. Am J Orthod dentofacial Orthop. 1996; 110: 423-430.

18. Ngan PW, Hagg U, Yiu C, Wei SH. Treatment response and long-term dentofacial adaptations to maxillary expansion and protraction. Semin Orthod 1997; 3:255-64.

19. Wolfe S; Araujo E; Behrents R; Buschang P.Craniofacial growth of Class III subjects six to sixteen years of age. Angle Orthod. 2011; 81:211-216.

20. Ngan P, Wei SH, Hagg U, Yiu CK, Merwin D, Stickel B. Effect of protraction headgear on Class III malocclusion. Quintessence Int. 1992; 23:197-207.

21. Kilicoglu H, Kirlic, Y. Profile changes in patients with Class III malocclusions after Delaire mask therapy. Am J Orthod Dentofacial Orthop. 1998; 113:453-462.

22. Yuksel S, Ucem TT, Keykubat A. Early and late facemask therapy. Eur J Orthod. 2001;23:559-568

23. Deguchi T, Kuroda T, Minoshima Y, Graber TM. Craniofacial features of patients with Class III abnormalities: growthrelated changes and effects of short-term and long-term chincup therapy. Am J Orthod Dentofacial Orthop. 2002; 121:84-92.

24. Baik HS, Jee SH, Lee KJ, Oh TK. Treatment effects of Frankel functional regulator III in children with Class III malocclusions. Am J Orthod Dentofacial Orthop. 2004; 125:294-301. 
25. Deguchi T, Kanomi R, Ashizawa Y, Rosenstein SW. Very early face mask therapy in Class III children. Angle Orthod. 1999; 69:349-355.

26. Masucci C, Franchi L, Defraia E, Mucedero M, Cozza P, Baccetti T. Stability of rapid maxillary expansion and facemask therapy: A long-term controlled study. Am J Orthod Dentofacial Orthop 2011; 140:493-500.

Recibido: 04-04-12

Aceptado: 21-06-12

Correspondencia: Álvarez-Emery, Universidad CES, Profesor Asistente de posgrado de Odontopediatría y Ortodoncia, Universidad CES/ ealvarez@ces.edu.co,Teléfono: 4440555 Ext 1515,Dirección: calle 10 A No. 22-04 Universidad CES, Medellín 